\title{
Measurement of Face Detection Accuracy Using Intensity Normalization Method and Homomorphic Filtering
}

\author{
I Nyoman Gede Arya Astawa ${ }^{[1] *}$, I Ketut Gede Darma Putra ${ }^{[2]}$, I Made Sudarma ${ }^{[3]}$, and Rukmi Sari Hartati ${ }^{[4]}$ \\ [1] Doctoral Programed of Engineering Science, Faculty of Engineering, Udayana University \\ Department of Electrical Engineering, Politeknik Negeri Bali, Indonesia \\ [2] Information Technology, Faculty of Engineering, Udayana University, Indonesia \\ [3][4] Electrical Engineering, Faculty of Engineering, Udayana University, Indonesia \\ *email : arya_kmg@pnb.ac.id
}

\begin{abstract}
One of the factors that affects the detection system or face recognition is lighting. Image color processing can help the face recognition system in poor lighting conditions. In this study, homomorphic filtering and intensity normalization methods used to help improve the accuracy of face image detection. The experimental results show that the nonuniform of the illumination of the face image can be uniformed using the intensity normalization method with the average value of Peak Signal to Noise Ratio (PSNR) obtained from the whole experiment is $\mathbf{2 2 . 0 5 3 1 4}$ and the average Absolute Mean Brightness Error (AMBE) value obtained is 6.147787. The results showed that homomorphic filtering and intensity normalization methods can be used to improve the detection accuracy of a face image.
\end{abstract}

Keywords: face detection, homomorphic filter, intensity normalization.

\section{INTRODUCTION}

Presently mobile device with an integrated digital camera has been widely used in obtaining a picture or image. Wherein, an image composed of a variety of information that makes it recognizable. Therefore, an image can be valuable information when processed in image recognition processes such as facial image. This was done because the facial image can provide specific information relating to personal identification, etc. [1].

This facial image recognition technology more widely applied in such fields as biometrics, search systems, and security systems. Where, in principle, this technology is compared with a rate of similarity of a face image. Face recognition technology is one of many research topics that is still being developed today. The topic has been studied for more than three decades. Today, the face recognition method has been developed and gives promising results [2].
One of the factors that affect the technology in the detection or face recognition is lighting [2]. Poor illumination can make the system does not recognize a face. Both the illumination is too dark or too bright. Color image processing can be done to help the multi-facial recognition system in poor lighting conditions, in order to be able to recognize the faces faster and more accurately.

In this study, homomorphic filtering and intensity normalization method based on mobile applications is applied. Use of these methods is to reduce noise [3] and reduce errors in detection of a face image [4].

This paper consisted of background research and related studies that have been done are described in the first and second parts. Homomorphic filtering and intensity normalization method described in the third section. As well, the results described in the fourth section.

\section{RESEARCH STUDY}

Until now, a lot of research being conducted in the area of facial recognition technology. Various methods have been used to detect a face image. Several studies have been conducted, among others, the use of homomorphic filtering methods on the images have been conducted by several researchers, among others, research utilizing methods Homomorphic Filtering on the images using three types of equations on the high-pass filter that is: Equation Butterworth, Equations Gaussian, and Equation exponent have been done. The experimental results obtained show that the homomorphic filtering method using the equation Butterworth has more advantages than 2 other equations [3]. Then, the face recognition research using Butterworth equation on the homomorphic filtering method has also been done. Meanwhile, the research results mention that homomorphic filtering can improve illumination without damaging detail and less noise [5]. 
Meanwhile, research that utilizes the intensity normalization method has also been conducted by several researchers such as the normalization method used to reduce the intensity of illumination uniformity in the image. The results showed that the intensity normalization method can be used to reduce the uniformity illumination $[4,6]$. Then, the normalization method is also used for image detection. The results showed that the uniformity illumination can affect the detection so that the value obtained an accuracy of $98.7 \%$. False Alarm Rate (FAR) and False Rejection Rate (FRR) has a value of less than $3 \%$. Likewise, the value of PSNR. This means that the lower AMBE value, the more uniform illumination at the second image. Method Peak Signal to Noise Ratio (PSNR) is used to calculate the value of contrast enhancement in the image. The higher the PSNR value, the higher the quality of the image $[4,7]$. After looking at the performance of some of the research above, the second-mentioned method will be used in this study.

\section{RESEARCH METHOD}

In this study, the color image processing conducted in steps. At the initial stage, the image acquired by the camera or an existing image. The image is then converted to $\mathrm{YCbCr}$ format. The second phase, after the image is converted, then the intensity normalization. The third phase, the conversion back to RGB format conducted so that each faces contained in the image can be detected. Face detection conducted on the image that has been normalized. The faces are detected from the image are taken. The fourth stage, the conversion to YCbCr performed on each detected face. Then, the face image noise is reduced by using homomorphic filtering method. Meanwhile, non-uniform illumination of the face image is uniformed by using intensity normalization method. The flow of the system can be seen in Fig. 1.

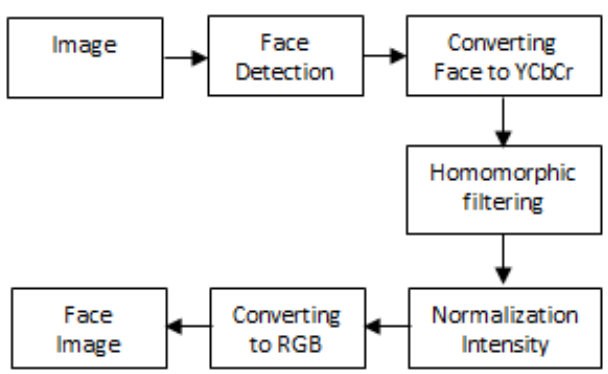

Figure 1 System flow

In this research, the image converted from RGB model to $\mathrm{YCbCr}$ because $\mathrm{YCbCr}$ color model is better for image compression and also $\mathrm{Y}$ Luminance can be processed separately [8]. Where,
Y Luminance is the gray scale of the image. As for, the conversion of the RGB color model to $\mathrm{YCbCr}$ is defined in Equation 1 [9].

$$
\left[\begin{array}{c}
\mathrm{Y} \\
\mathrm{Cb} \\
\mathrm{Cr}
\end{array}\right]=\left[\begin{array}{ccc}
0.229 & 0.587 & 0.114 \\
-0.169 & -0.331 & 0.500 \\
0.500 & -0.419 & -0.081
\end{array}\right] \times\left[\begin{array}{c}
\mathrm{R} \\
\mathrm{G}+128 \\
\mathrm{~B}+128
\end{array}\right]
$$

Meanwhile, the intensity normalization method in the image is used to reduce the non-uniform of illumination in the image [10]. As for, the formula used for the intensity normalization is the same as that done by [6] in his research. The formula can be seen in Equations 2 and 3.

$$
\begin{aligned}
& g(x, y)=\left\{\begin{array}{cr}
\phi_{d}+\gamma, & \text { if } \mathrm{f}(\mathrm{x}, \mathrm{y})>\phi \\
\phi_{\mathrm{d}}-\gamma, & \text { otherwise }
\end{array}\right. \\
& \gamma=\sqrt{\frac{\rho_{\mathrm{d}}\{\mathrm{f}(\mathrm{x}, \mathrm{y})-\phi\}^{2}}{\rho}}
\end{aligned}
$$

Where, $\mathrm{f}$ and $\mathrm{g}$ respectively express the image before and after normalized, $\phi$ and $\rho$ denote mean and variance value of the image $\phi_{d}$ and $\rho_{d}$ are desired mean and variance value.

In this study, the process of homomorphic filtering method use Butterworth equation. The process steps of Homomorphic Filtering begin with the transformation of logarithm (ln), then fast Fourier (FFT), filtering $(\mathrm{H}(\mathrm{u}, \mathrm{v}))$, inverse fast fourier (IFFT), and exponential (exp) transformations. Overview for homomorphic filtering process can be seen in Figure 2 . Meanwhile, High Pass Butterworth technique uses Equation 4.

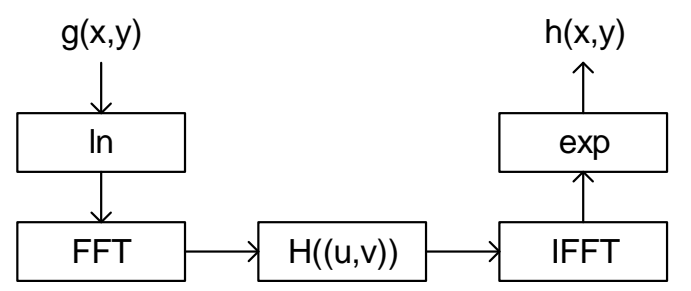

Figure 2 Homomorphic filtering overview process

$$
H(u, v)=\frac{1}{1+\left(D_{0} / D(u, v)\right)^{2 n}}
$$

Where, the notation $\mathrm{D}(\mathrm{u}, \mathrm{v})$ is the distance from the fourier transform centered origin; $D_{0}$ is the cutoff distance measured from its origin; and $n$ notation is a butterworth sequence [11].

The next process is normalization and filtering where the image is converted back into the RGB color model. Convert YCbCr color model back to RGB color model using Equation 5 [12]. 


$$
\left[\begin{array}{l}
\mathrm{R} \\
\mathrm{G} \\
\mathrm{B}
\end{array}\right]=\left[\begin{array}{ccc}
1 & 0 & 1.402 \\
1 & -0.344 & -0.714 \\
1 & 1.772 & 0
\end{array}\right] \times\left[\begin{array}{c}
\mathrm{Y} \\
\mathrm{Cb}-128 \\
\mathrm{Cr}-128
\end{array}\right]
$$

\section{A. Datasets}

In this study, the datasets are images that contain many faces. Where, the image is taken from social media, digital camera, and mobile phone. As for, the device used for this experiment is the Samsung Galaxi tab. Examples of normalized image detection and comparison can be seen in Figure 3.

\section{IV.DISCUSSION}

This section describes the experimental results with the method of homomorphic filtering method and the intensity normalization method.

Based on Figure 3 (a) shows the face image before it is normalized. Where, only 12 faces detected. Whereas, (b) shows that 14 face images can be detected. After the image is normalized, then done crop on each - each face image. Furthermore, the face image is processed by using homomorphic filtering method and intensity normalization method. Six faces of the processed face image can be seen in Figure 4

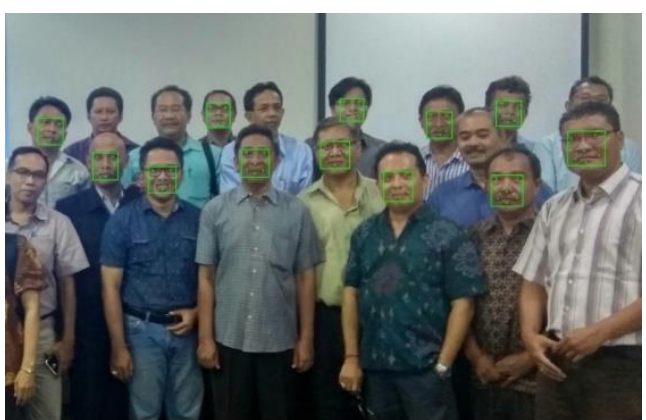

(a)

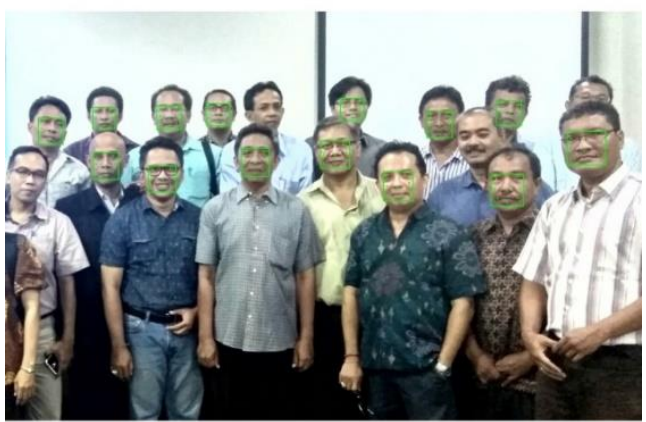

(b)

Figure 3 Comparison of detected faces before and after normalization. (a) image before normalization, (b) image after normalization
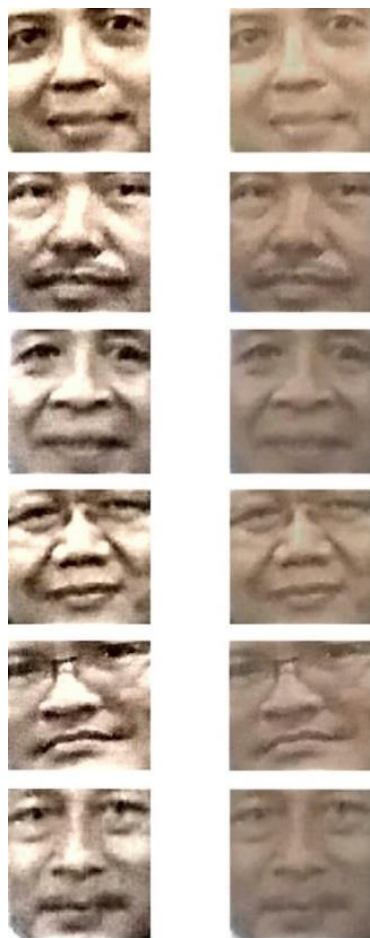

(a)

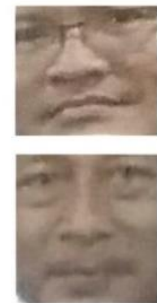

(b)
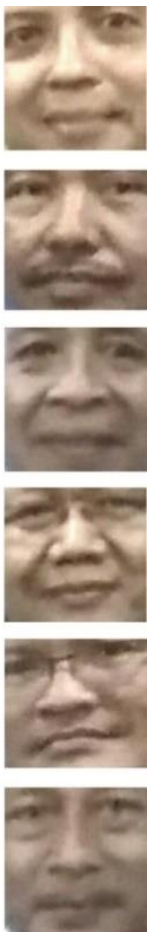

(c)

Figure 4 An example of a processed face. (a) face before being processed. (b) the face has been processed with homomorphic filtering. (c) face after homomorphic filtering process + intensity normalization

The experimental results, in Figure 4, show that the image color is smoother than before being processed. Meanwhile, the comparison of the level of illumination on the processed face is used the Absolute Mean Brightness Error (AMBE) method. AMBE is used to calculate mean brightness difference between 2 images. Meanwhile, the average Absolute Mean Brightness Error (AMBE) obtained is 6.147786761. Meanwhile, the average value of Peak Signal to Noise Ratio (PSNR) obtained from the overall experiment was 22.053. The average table of PSNR and AMBE values from each experiment can be seen in Table 1 .

TABLE 1 PSNR AND AMBE AVERAGE VALUE

\begin{tabular}{|c|l|l|l|l|l|}
\hline PSNR & AMBE & $\begin{array}{c}\text { Va- } \\
\text { riance }\end{array}$ & Mean & Source & $\begin{array}{c}\text { Number } \\
\text { of data }\end{array}$ \\
\hline 21.92977 & 6.173859 & 180 & 150 & $\begin{array}{l}\text { Social } \\
\text { media } \\
\text { Social } \\
\text { media }\end{array}$ & 105 \\
22.42403 & 5.965482 & 150 & 150 & $\begin{array}{l}\text { Digital } \\
\text { Camera }\end{array}$ & 105 \\
21.20388 & 5.867629 & 150 & 150 & $\begin{array}{l}\text { Digital } \\
\text { Camera } \\
\text { Mobile } \\
\text { phone } \\
\text { Mobile } \\
\text { phone }\end{array}$ & 105 \\
22.73389 & 6.687012 & 180 & 150 & 150 & 105 \\
23.17672 & 6.42274 & 150 & 150 & \multicolumn{3}{|l|}{ average } \\
\hline 22.05314 & 6.147787 & \multicolumn{3}{|c|}{150} & \\
\hline
\end{tabular}


In this experiment, variant value of the source image are 180 and 150 for each source. Where, each source uses 105 images. This shows that face images had taken from various sources: social media, digital camera, and mobile phone can be processed by using method of homomorphic filtering and intensity normalization method.

\section{CONCLUSION}

Performance of color image processing using intensity normalization and homomorphic filtering methods in this study obtain an average value of Peak Signal to Noise Ratio (PSNR) obtained from the whole trial is equal to 22.05314 and the average value of Absolute Mean Brightness Error (AMBE) obtained is 6.147787. Best AMBE value that obtained was from digital camera is 5.77 and the best PSNR obtained from mobile phone amounted to 23.177. Face detection accuracy has also increased when the intensity normalization method implemented in the image. The results showed that the method of homomorphic filtering and intensity normalization can be used to improve the accuracy of detection of a face image.

\section{REFERENCES}

[1] P. Peng and Y. Shen, "Efficient face verification in mobile environment using component-based PCA," in 2013 6th International Congress on Image and Signal Processing (CISP), 2013, pp. 753-757.

[2] X. Jiang, D. Zhang, and X. Feng, "Local feature hierarchy for face recognition across pose and illumination," in 2016 Sixth International Conference on Image Processing Theory, Tools and Applications (IPTA), 2016, pp. 1-5.
[3] L. Xiao, C. Li, Z. Wu, and T. Wang, "An enhancement method for X-ray image via fuzzy noise removal and homomorphic filtering," Neurocomputing, vol. 195, pp. 56-64, 2016

[4] I. K. G. D. Putra, W. Bhuana, and Erdiawan, "Pembentukan Kode Telapak Tangan (Palm Kode) Berbasis Metode GABOR 2D," MAKARA, Teknologi, vol. 15, pp. 161-167, November 2011

[5] H. Shahamat and A. Pouyan, "Face recognition under large illumination variations using homomorphic filtering in spatial domain," Journal of Visual Communication and Image Representation, vol. 25, pp. 970-977, 2014.

[6] A. Kumar, D. C. M. Wong, H. C. Shen, and A. K. Jain, "Personal verification using palmprint and hand geometry biometric," presented at the Proceedings of the 4th international conference on Audio- and video-based biometric person authentication, Guildford, UK, 2003.

[7] B. Gupta and M. Tiwari, "Minimum mean brightness error contrast enhancement of color images using adaptive gamma correction with color preserving framework," Optik - International Journal for Light and Electron Optics, vol. 127, pp. 1671-1676, 2016.

[8] N. A. Ibraheem, M. M. Hasan, R. Z. Khan, and P. K. Mishra, "Understanding Color Models: A Review," ARPN Journal of Science and Technology, vol. 2, pp. 265-275, 2012.

[9] N. K. Patil, Y. R. M., and J. Pujari, "Comparison Between HSV and $\mathrm{YCbCr}$ Color Model Color-Texture based Classification of the Food Grains," International Journal of Computer Applications, vol. 34, pp. 51-7, 2011.

[10] H. Lin, W. Yifei, and A. Jain, "Fingerprint image enhancement: algorithm and performance evaluation," IEEE Transactions on Pattern Analysis and Machine Intelligence, vol. 20, pp. 777-789, 1998.

[11] Z. Zhong and Z. Geng, "Butterworth filter and Sobel edge detection to image," in 2011 International Conference on Multimedia Technology, 2011, pp. 254-256.

[12] Y. Yang, P. Yuhua, and L. Zhaoguang, "A Fast Algorithm for $\mathrm{YCbCr}$ to RGB Conversion," IEEE Transactions on Consumer Electronics, vol. 53, pp. 14901493, 2007. 